PPPL-3451

PPPL-3451

UC-70

Drift Mode Calculations for the Large Helical Device

by

G. Rewoldt, L.-P. Ku, W.M. Tang, H. Sugama, N. Nakajima, K.Y. Watanabe, S. Murakami, H. Yamada, and W.A. Cooper

June 2000
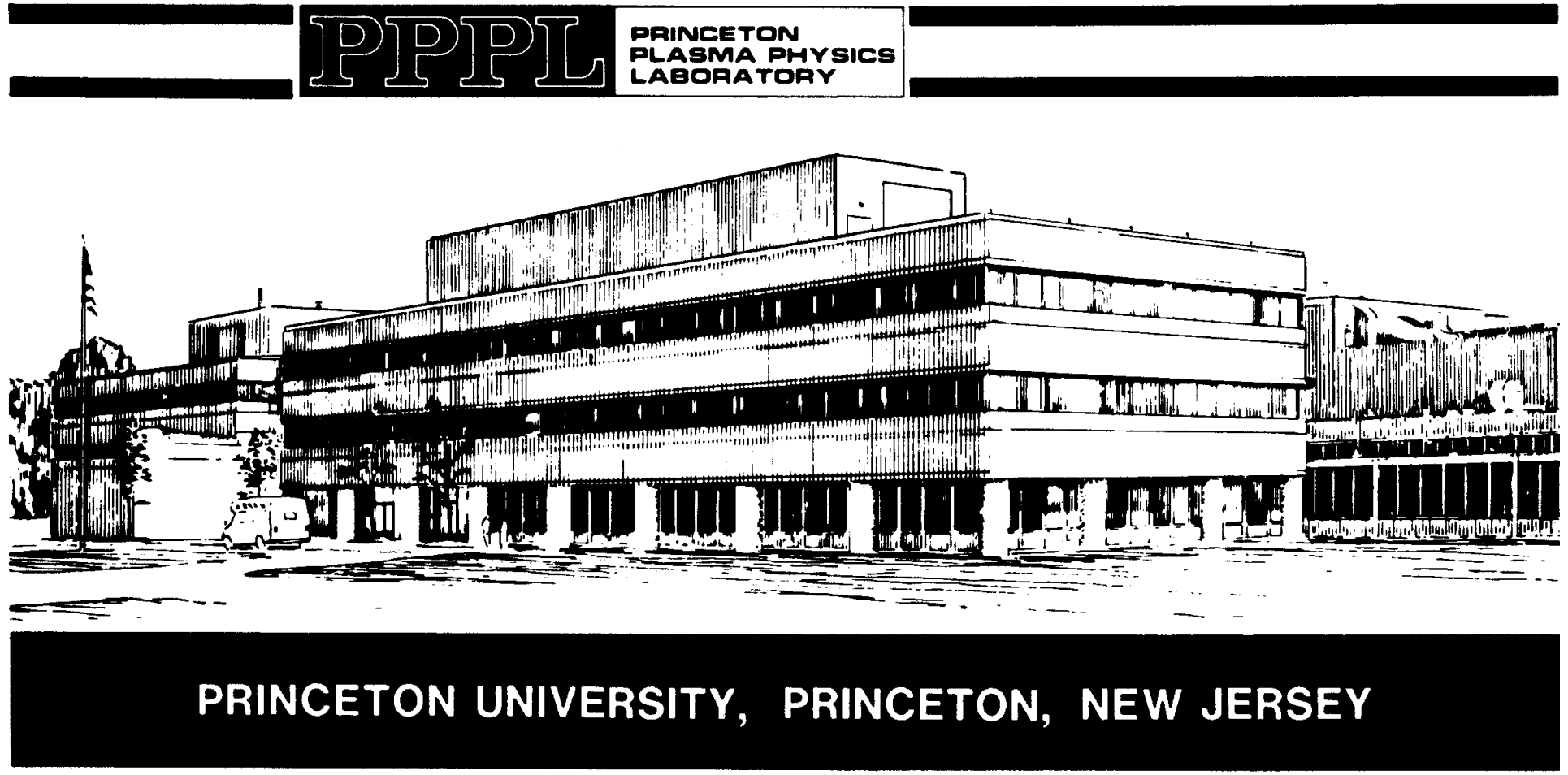


\section{PPPL Reports Disclaimer}

This report was prepared as an account of work sponsored by an agency of the United States Government. Neither the United States Government nor any agency thereof, nor any of their employees, makes any warranty, express or implied, or assumes any legal liability or responsibility for the accuracy, completeness, or usefulness of any information, apparatus, product, or process disclosed, or represents that its use would not infringe privately owned rights. Reference herein to any specific commercial product, process, or service by trade name, trademark, manufacturer, or otherwise, does not necessarily constitute or imply its endorsement, recommendation, or favoring by the United States Government or any agency thereof. The views and opinions of authors expressed herein do not necessarily state or reflect those of the United States Government or any agency thereof.

\section{Availability}

This report is posted on the U.S. Department of Energy's Princeton Plasma Physics Laboratory Publications and Reports web site in Calendar Year 2000. The home page for PPPL Reports and Publications is: http://www.pppl.gov/pub_report/

DOE and DOE Contractors can obtain copies of this report from:

U.S. Department of Energy

Office of Scientific and Technical Information

DOE Technical Information Services (DTIS)

P.O. Box 62

Oak Ridge, TN 37831

Telephone: (865) 576-8401

Fax: (865) 576-5728

Email: reports@adonis.osti.gov

This report is available to the general public from:

National Technical Information Service

U.S. Department of Commerce

5285 Port Royal Road

Springfield, VA 22161

Telephone: $1-800-553-6847$ or

(703) $605-6000$

Fax: (703) 321-8547

Internet: http://www.ntis.gov/ordering.htm 


\title{
Drift Mode Calculations for the Large Helical Device
}

\author{
G. Rewoldt, L.-P. Ku, and W. M. Tang \\ Plasma Physics Laboratory, Princeton University, \\ Princeton, New Jersey 08543-0451
}

H. Sugama, N. Nakajima, K. Y. Watanabe, S. Murakami, and H. Yamada National Institute for Fusion Science, Toki 509-5292, Japan

\author{
W. A. Cooper \\ Centre de Recherche en Physique des Plasmas, Ecole Polytechnique Fédérale de Lausanne, \\ PPB Ecublens, CH-1015 Lausanne, Switzerland
}

\begin{abstract}
A fully kinetic assessment of the stability properties of toroidal drift modes has been obtained for a case for the Large Helical Device (LHD) [A. Iiyoshi, et al., Plasma Physics and Controlled Nuclear Fusion Research, 1998, Nucl. Fusion 39, 1245 (1999)]. This calculation retains the important effects in the linearized gyrokinetic equation, using the lowest-order "ballooning representation" for high toroidal mode number instabilities in the electrostatic limit. Results for toroidal drift waves destabilized by trapped particle dynamics and ion temperature gradients are presented, using three-dimensional magnetohydrodynamic equilibria reconstructed from experimental measurements. The effects of helically-trapped particles and helical curvature are investigated. $52.35 \mathrm{Qz}, 52.65 \mathrm{Tt}, 52.55 \mathrm{Hc}$
\end{abstract}

Typeset using REVTEX 


\section{INTRODUCTION}

Drift mode calculation results are presented here for a case representing the Large Helical Device $^{1}$ (LHD), specifically for discharge 2900 at $t=0.6 \mathrm{~s}$. The temperature profiles and the density profile used were published previously in Ref. 2. Using this and other experimental information, a reconstructed magnetohydrodynamic (MHD) equilibrium is calculated using the three-dimensional VMEC equilibrium code, ${ }^{3,4}$ in VMEC coordinates. The MHD equilibrium is transformed to Boozer coordinates ${ }^{5}$ by the TERPSICHORE code, ${ }^{6}$ and needed data along a chosen magnetic field line for the instability calculation in FULL is constructed by the VVBAL code. ${ }^{7}$ The linear kinetic microinstability calculation is performed by the FULL code. The original tokamak (axisymmetric) version of this code is described in Refs. 8 and 9. The extension of this code to the stellarator (nonaxisymmetric) case and results for a quasiaxisymmetric case generated as part of the design process for the National Compact Stellarator Experiment ${ }^{10,11}$ (NCSX) are described in Ref. 12. The calculation and code employed here are identical to the ones described in that reference.

The nonaxisymmetric version of the FULL code retains the dynamics of the axisymmetric version of the code, in the electrostatic limit. It is a high- $n$ (toroidal mode number) calculation employing the lowest-order ballooning representation. It includes trapped particles, finite Larmor radius effects to all orders, banana orbital dynamics, bounce and transit and magnetic drift frequency resonances, equilibrium shaping effects, etc., for all species. Particle turning points are found numerically along the magnetic field line for each pitch angle, so that all classes of trapped particles are included automatically, including both toroidally-trapped and helically-trapped particles. The same extended form of model Krook collision operator described in Ref. 12 is employed here, which allows for multiple classes of trapped particles.

The previous quasiaxisymmetric case results in Ref. 12 were in a sense rather tokamaklike, in that the fraction of helically-trapped particles was small, and they played only a small role in the mode dynamics, compared to the toroidally-trapped particles. In the 
present LHD case, the helical magnetic field wells (along the magnetic field line) are much larger, so that there is a much larger fraction of helically-trapped particles, and they and the helical curvature can affect the mode dynamics much more strongly. Also, in the present LHD case, the MHD equilibrium and the density and temperature profiles are experimentally derived, rather than being somewhat arbitrarily chosen as in the quasiaxisymmetric case. In addition, only electron and background ion species were included for the quasiaxisymmetric case, whereas three impurity species, as well as a hot beam particle species with a slowingdown equilibrium distribution function, are also included for the LHD case. Thus, the present LHD case is in a sense more realistic than the previous quasiaxisymmetric case.

The LHD case is described and results for variation of a number of parameters in the collisionless limit are presented in Sec. II. Collisional effects are investigated and results of a radial scan are presented in Sec. III. Conclusions are given in Sec. IV.

\section{COLLISIONLESS RESULTS}

For LHD discharge 2900 at $t=0.6 \mathrm{~s}$, the helical coil center major radius is $R=3.9 \mathrm{~m}$, the average plasma minor radius $a=0.6 \mathrm{~m}$, the magnetic field $B_{0}=1.5 \mathrm{~T}$, the magnetic axis major radius is at $R_{m a}=3.75 \mathrm{~m}$, representing an inward shift, and the average elongation is one, with no ohmic current. At this time, the plasma is in a low-recycling pump-out phase. The background ion and hot beam ion species are both hydrogen, and the observed impurities are carbon $(C)$, oxygen $(O)$, and iron $(F e)$ in the estimated ratios 1:1:0.5, with an estimated $Z_{e f f}=2$, so that we take $n_{C} / n_{e}=n_{O} / n_{e}=2 n_{F e} / n_{e}=0.0024$ at all radii. Thus, six species in all are included in the FULL code calculation. The beam species density profile is calculated using a three-dimensional Monte Carlo simulation code. ${ }^{13}$ A slowingdown equilibrium distribution function is used for the hot beam species, and Maxwellian distributions for all of the other species. The electron and ion temperature profiles and the electron density profile shown in Fig. 3 of Ref. 2 are used here in modified form.

We first consider the magnetic surface $s=0.875 \simeq(\mathrm{r} / \mathrm{a})^{2}$, with the magnetic field line 
in this surface specified by $\alpha \equiv \zeta-q \theta=0$ and the ballooning parameter $\theta_{0}=0\left(\theta_{0}\right.$ is sometimes called $\theta_{k}$ ). Each of these parameters will be varied individually later in this section. The ballooning representation (nonperiodic) input functions on this magnetic field line are shown in Fig. 1 for $B(\theta) \equiv|\mathbf{B}(\theta)|$, in Fig. 2 for $k_{\perp}^{2}(\theta) / n^{2}$, and in Fig. 3 for the curvature drift $\equiv \mathbf{k}_{\perp} \cdot\{\mathbf{b} \times[(\mathbf{b} \cdot \nabla) \mathbf{b}]\} / n$. Here, $\theta$ (poloidal angle) is used as the nonperiodic ballooning coordinate along the magnetic field line. Data is used over $252 \pi$ periods in $\theta$, from $-12.5 \times 2 \pi$ to $12.5 \times 2 \pi$, but only data for the center period, from $-\pi$ to $\pi$, is shown in these figures. Each of these input functions is symmetric (even) in $\theta$ around $\theta=0$, when $\theta_{0}=\alpha=0$. This is in a sense a symmetry point of the MHD equilibrium. In this situation, the instability eigenfunctions are either symmetric (even) or antisymmetric (odd) in $\theta$ around $\theta=0$. However, when either $\theta_{0} \neq 0$ or $\alpha \neq 0$ (or some other symmetry values of $\theta_{0}$ and $\alpha$ ), all of these symmetries are broken.

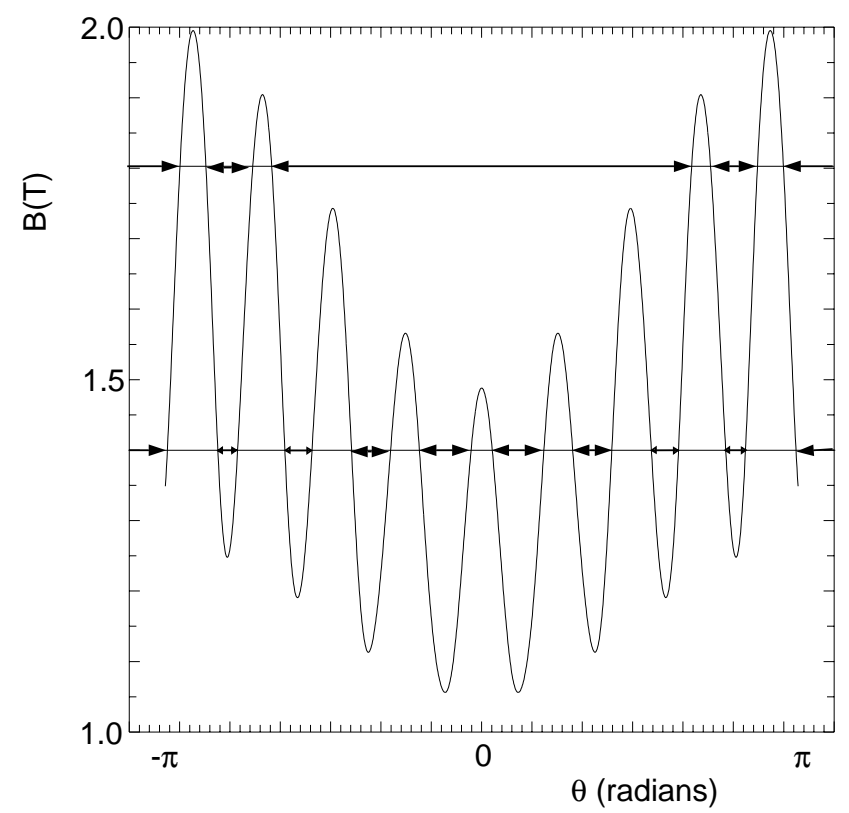

FIG. 1. Variation of $B(\theta)$ along chosen magnetic field line with $\alpha=0$ and $\theta_{0}=0$, on magnetic surface 4.9 of 61 ( $s=0.825)$, for case for LHD discharge 2.900 at $t=0.6 s$.

For the magnetic field strength shown in Fig. 1, the short-scale helical variation is comparable in amplitude to the longer-scale toroidal variation. For the illustrative pitch angle corresponding to $B=1.8 \mathrm{~T}$, there is one class of toroidally-trapped particles and four classes 


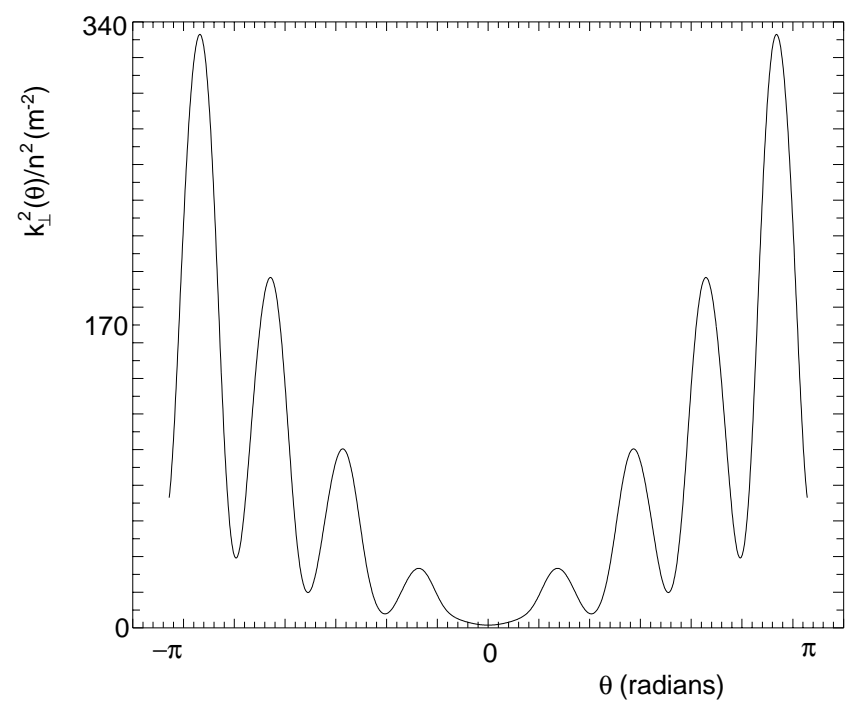

FIG. 2. Variation of $k_{\perp}^{2}(\theta) / n^{2}$ along chosen magnetic field line, for case of Fig. 1 .

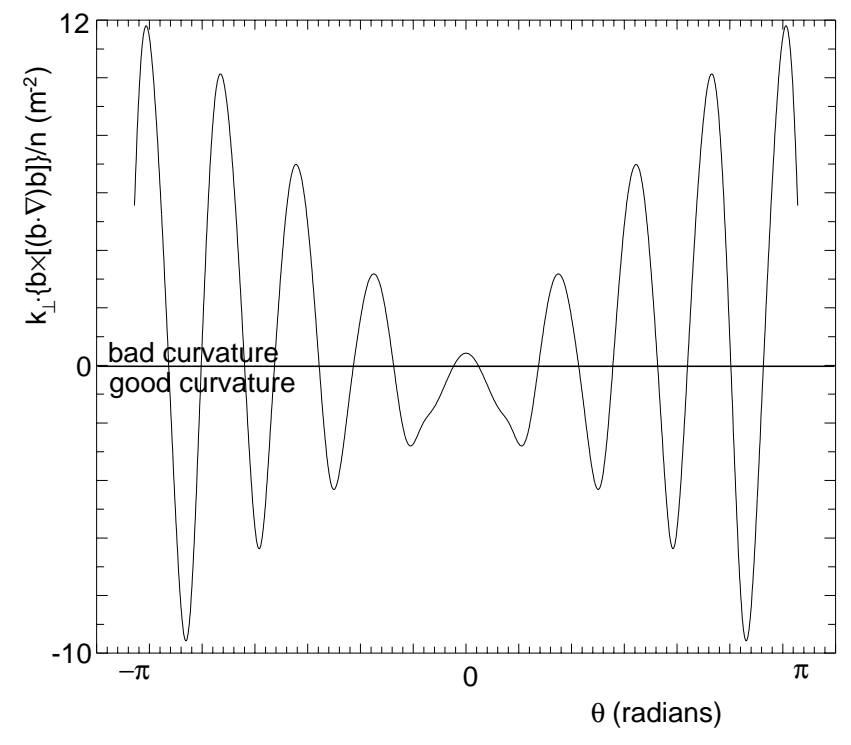

FIG. 3. Variation of curvature drift $\mathbf{k} \cdot\{\mathbf{b} \times[(\mathbf{b} \cdot \nabla) \mathbf{b}]\} / n$ along chosen magnetic field line, for case of Fig. 1. 
of helically-trapped particles with one or both turning points in this center period. For the illustrative pitch angle corresponding to $B=1.4 \mathrm{~T}$, there are ten trapped-particle classes with one or both turning points in this center period, which cannot be conveniently classified as either toroidally or helically trapped. All of the trapped particle classes in all of the periods are found numerically by the FULL code and used in its kinetic calculation.

The eigenfunction for the perturbed electrostatic potential $\hat{\phi}(\theta)$ for an unstable mode, for the experimental values $\eta_{i}^{e} \equiv d \ln T_{i} / d \ln n_{e}=0.80$ and $\eta_{e} \equiv d \ln T_{e} / d \ln n_{e}=0.85$, for $k_{\perp} \rho_{i}(\theta=0)=0.56$ and $n=237$, without collisions, is shown in Fig. 4 . The parameters that have been mentioned so far are our basic case parameters, which we will vary one by one later in this section. This eigenfunction is even in $\theta$ around $\theta=0$. Odd eigenfunctions in $\theta$ can also be found, as will be seen. The eigenfunction in Fig. 4 is mainly localized within the toroidal magnetic field well around $\theta=0$, and strongly overlaps only a few helical magnetic field wells.

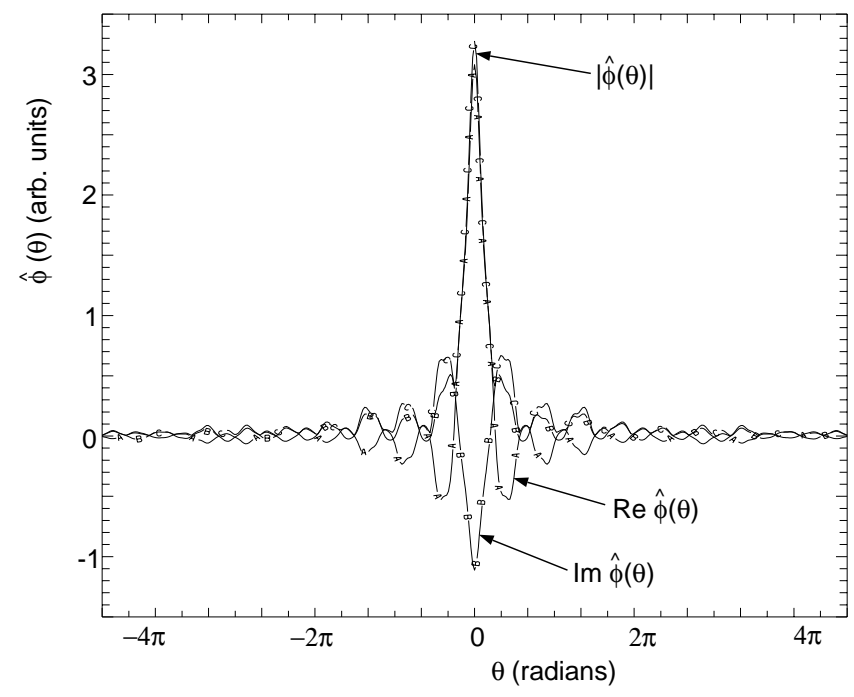

FIG. 4. Eigenfunction of electrostatic, collisionless toroidal drift mode (TEM root) along chosen magnetic field line, for case of Fig. 1 , with $\eta_{i}^{e}=0.80, \eta_{e}=0.85$, and $k_{\perp} \rho_{i}(\theta=0)=0.56(n=237)$, including electrons, hydrogen background ions, carbon, oxygen, and iron impurity species, and a hydrogen hot beam species with a slowing-down distribution.

The eigenfrequency corresponding to this eigenfunction is $\omega=(+2.3+4.9 i) \times 10^{5} \mathrm{~s}^{-1}$, 
with positive real frequencies meaning: in the electron diamagnetic direction. This root will be labeled as a trapped-electron mode (TEM) root, because of its characteristics when $\eta_{i}^{e}$ and $\eta_{e}$ are varied artificially. There is another root labeled as an ion temperature gradient mode (ITG) root that is only unstable for $\eta_{i}^{e}=\eta_{e}$ above a critical value $\eta_{c}=1.35$, with the other basic case parameters, and has a real frequency in the ion diamagnetic direction. (The ITG root would be part of the "toroidicity-induced branch", in tokamak nomenclature.) Both of these roots have eigenfunctions that are even in $\theta$. This ITG root is stable for the experimental values of $\eta_{i}^{e}=0.80$ and $\eta_{e}=0.85$. This behavior is shown in Fig. 5 , where $\eta_{i}^{e}=$ $\eta_{e}$ is varied artificially at fixed total pressure gradient, so that $d T_{i} / d r$ and $d T_{e} / d r$ increase and $d n_{e} / d r$ decreases as $\eta_{i}^{e}=\eta_{e}$ increases. For this particular case, the respective real frequencies $\omega_{r}$ never change sign. The TEM root growth rate $\gamma$ is monotonically decreasing in $\eta_{i}^{e}=\eta_{e}$, while that of the ITG root is monotonically increasing, with a crossover at about $\eta_{i}^{e}=\eta_{e}=2.5$. For this case, the TEM root and the ITG root are separate roots, and do not hybridize into a single root, as often happens in tokamak cases.

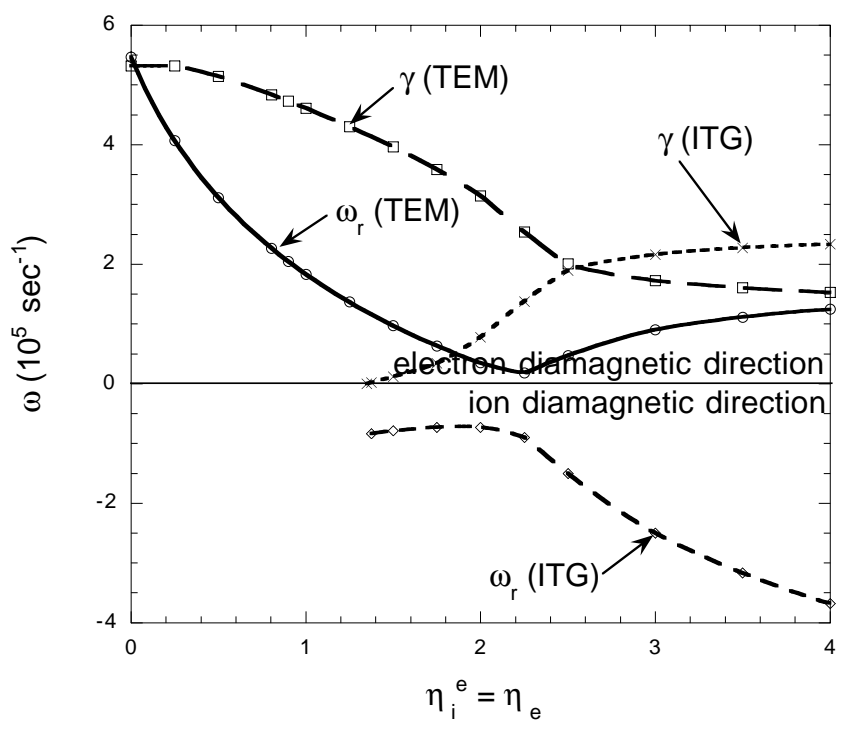

FIG. 5. Variation of linear growth rate $\gamma$ and real frequency $\omega_{r}$ for TEM and ITG roots with $\eta_{i}^{e}=\eta_{e}$, for $k_{\perp} \rho_{i}(\theta=0)=0.56(n=237)$. Here, the total pressure gradient is held fixed as $\eta_{i}^{e}=\eta_{e}$ varies.

The effect of the beam species is slightly stabilizing for the TEM root for this case, and 
that of the impurities is very slightly destabilizing, for the basic case parameters. Turning off the beam species (i.e., replacing the beam particles with additional background ion particles so that $n_{e}$ is unchanged), the value of $\omega$ for the TEM root with the experimental values of $\eta_{i}^{e}$ changes to $\omega=(+2.5+5.5 i) \times 10^{5} \mathrm{~s}^{-1}$, and with all three impurity species also turned off in the same way, it becomes $\omega=(+2.0+5.3 i) \times 10^{5} \mathrm{~s}^{-1}$. For the ITG root at $\eta_{i}^{e}=\eta_{e}=4.0$, with the other basic case parameters, the effect of the beam species is essentially neutral, and that of the three impurity species is very slightly stabilizing. With all six species included, the ITG root eigenfrequency for $\eta_{i}^{e}=\eta_{e}=4.0$ is $\omega=(-3.8+2.3 i) \times 10^{5} \mathrm{~s}^{-1}$, while with the beam species turned off it is $\omega=(-3.8+2.3 i) \times 10^{5} \mathrm{~s}^{-1}$, and with all three impurity species also turned off it is $\omega=(-4.3+2.4 i) \times 10^{5} \mathrm{~s}^{-1}$. For other cases, the effects of the beam species and the impurity species could be much stronger than is seen for this case.

The variation of $\gamma$ and $\omega_{r}$ with $k_{\perp} \rho_{i}(\theta=0)$ or $n$ is shown in Fig. 6 for the TEM root, with the other basic parameters. The growth rate peaks for $k_{\perp} \rho_{i}(\theta=0) \simeq 0.5$ to 0.6 , and the change of the real frequency is from the electron towards the ion diamagnetic direction as $k_{\perp} \rho_{i}(\theta=0)$ increases. This is the kind of $k_{\perp} \rho_{i}$ dependence that is familiar for tokamaks. However, the TEM root real frequency stays in the electron diamagnetic direction over this range.

The effect on $\gamma$ and $\omega_{r}$ of varying the ballooning parameter $\theta_{0}$ (sometimes called $\theta_{k}$ ) through one period for the TEM root (which has an even eigenfunction in $\theta$ around $\theta=0$ for $\theta_{0}=\alpha=0$ ) for the other basic parameters is shown in Fig. 7. Also shown is another root, which corresponds to an eigenfunction which is odd in $\theta$ around $\theta=0$ for $\theta_{0}=\alpha=0$. We will refer to this second root generally as the odd root, even though the eigenfunction has odd symmetry only for $\theta_{0}=\alpha=0$. Antisymmetric modes have been investigated previously for tokamaks in a more approximate way, for instance in Ref. 14. The growth rate of the odd root is much less than that of the TEM root for any value of $\theta_{0}$. The effect on $\gamma$ and $\omega_{r}$ of varying the field line label $\alpha$ through one period on these two roots for the other basic parameters is shown in Fig. 8 . The growth rate of the odd root is much less than that of the TEM root for $\alpha=0$, but becomes greater than that of the TEM root for $\alpha=\pi / 10$ (i.e., 


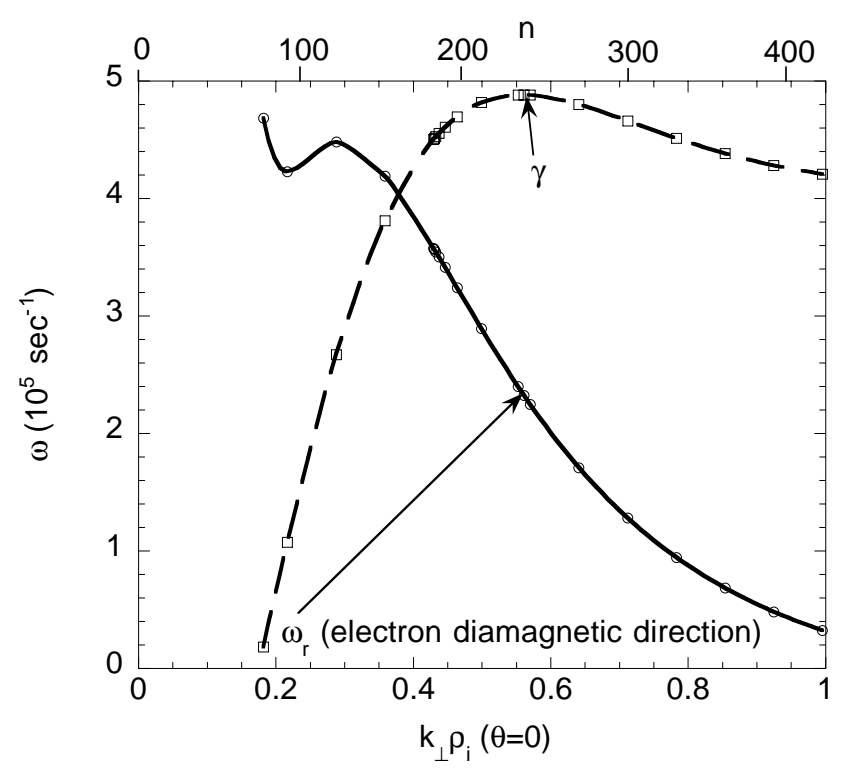

FIG. 6. Variation of $\gamma$ and $\omega_{r}$ with $k_{\perp} \rho_{i}(\theta=0)$ and $n$, for case of Fig. 4 for TEM root.

halfway through one period of the ten toroidal period LHD). However, the highest growth rate overall occurs for the TEM root at $\theta_{0}=\alpha=0$.

By comparing the present case with an artificial case in which all of the helical components are set to zero in the VVBAL code in evaluating the input functions along the magnetic field line for the FULL code, the effect of the helically-trapped particles and the helical curvature is seen to be strongly destabilizing for the TEM root and slightly stabilizing for the ITG root. For the TEM root with the basic parameters, so that $\eta_{i}^{e}=0.80$ and $\eta_{e}=0.85$, the eigenfrequency with the helical contributions suppressed is $\omega=(+1.1+0.7 i) \times 10^{5} \mathrm{~s}^{-1}$, while with the helical contributions included it is $\omega=(+2.3+4.9 i) \times 10^{5} \mathrm{~s}^{-1}$, a substantial increase in the growth rate. For this value of $\eta_{i}^{e}$, the TEM root is destabilized mainly by the collisionless trapped-electron mode mechanism, which is a toroidal magnetic drift precession frequency resonance mechanism entering through the trapped-particle time-average term. ${ }^{8}$ Suppressing the helically-trapped particles and helical curvature decreases the amount of "bad" curvature available for this destabilization. For the ITG root with $\eta_{i}^{e}=\eta_{e}=4.0$, the eigenfrequency with the helical contributions suppressed is $\omega=(-2.1+2.8 i) \times 10^{5} \mathrm{~s}^{-1}$, while with the helical contributions included it is $\omega=(-3.8+2.3 i) \times 10^{5} \mathrm{~s}^{-1}$, a moderate 


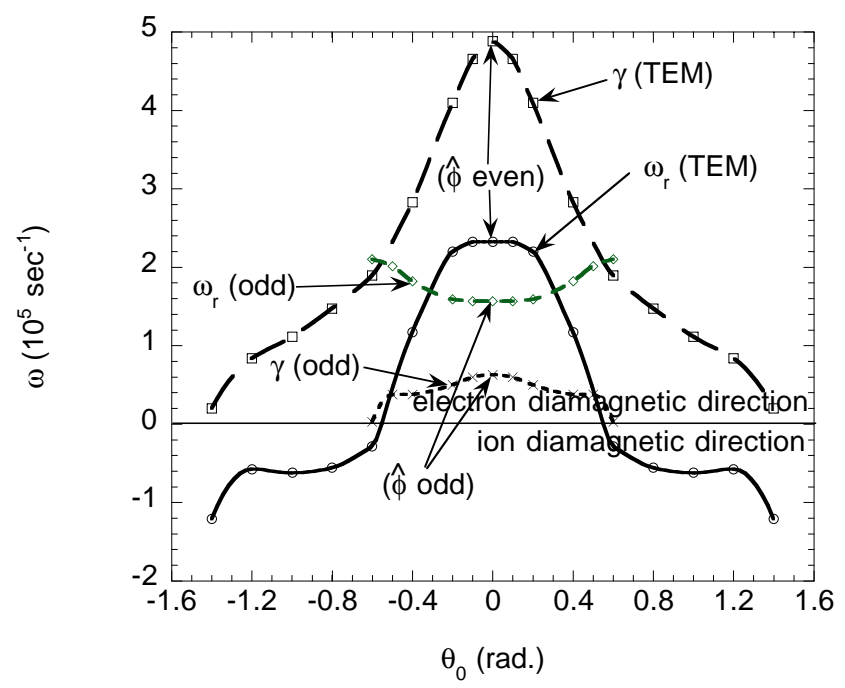

FIG. 7. Variation of $\gamma$ and $\omega_{r}$ with ballooning parameter $\theta_{0}$, for TEM and odd roots, for case of Fig. 4, with $\alpha=0$.

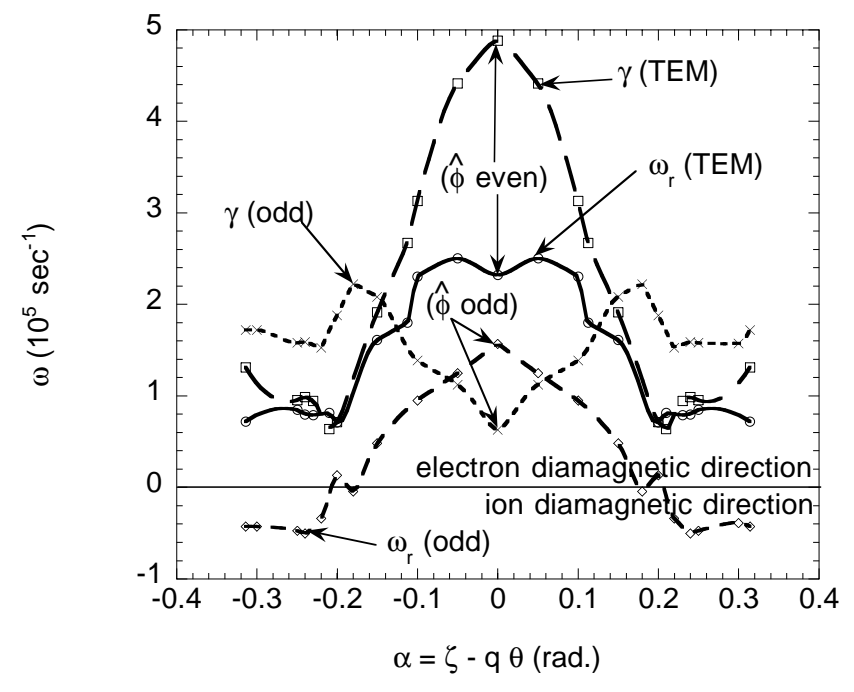

FIG. 8. Variation of $\gamma$ and $\omega_{r}$ with magnetic field line label $\alpha \equiv \zeta-q \theta$, for TEM and odd roots, for case of Fig. 4, with $\theta_{0}=0$. 
decrease in the growth rate. At this $\eta_{i}^{e}=\eta_{e}$ value, the ITG root is destabilized mainly by the ion temperature gradient mechanism entering through the ion terms, ${ }^{8}$ which does not require "bad" curvature to be effective, but only requires that the ion temperature gradient be larger than a critical value. However, the collisionless trapped electron mode destabilization mechanism can still make a contribution at any value of $\eta_{i}^{e}$. For other cases, the contribution of the helically-trapped particles and the helical curvature could in general be either destabilizing or stabilizing. Note that this comparison is not the same as a comparison to a tokamak case, because the present artificial case without the helical contributions still has the opposite sign of the (average) magnetic shear everywhere from that of a standard tokamak, among other differences. In particular, negative magnetic shear does not have to affect the helical curvature in the same way as the toroidal curvature.

\section{COLLISIONAL EFFECTS}

The nonaxisymmetric version of the FULL code uses a model Krook collision operator that is described in more detail in Ref. 12. Briefly, for axisymmetric geometry it models the two-step physical process of detrapping and retrapping, which gives an effective collision frequency which diverges at the trapped-passing boundary. This approach gives good agreement with results from an equivalent calculation using a Lorentz pitch-angle scattering operator, in a tokamak comparison. ${ }^{15}$ In the nonaxisymmetric version, where there are multiple classes of trapped particles, the model collision operator is generalized to include transitions between adjacent trapped-particle classes also. The nonaxisymmetric FULL code finds all local maxima of $B(\theta)$, and the effective collision frequency is constructed so as to diverge (in pitch angle) at these maxima, modeling the behavior of a Lorentz collision operator.

Note that the pressure and pressure gradient are input to the FULL code through the MHD equilibrium. The total pressure gradient is allocated to the density and temperature gradients of the individual species using the eta's, the density fractions, the temperatures, 
etc., of the individual species. The separate electron density that is input is used only to calculate the collision frequencies. Thus, a zero electron density specifies the collisionless limit, but does not affect the rest of the calculation.

The behavior of $\gamma$ and $\omega_{r}$ with the electron density $n_{e}$ and with the usual tokamak collisionality parameter $\nu_{e}^{*}$ is shown in Fig. 9 for the TEM root for the basic case parameters. At the experimental values of $n_{e}$ and $\nu_{e}^{*}$, there is a substantial reduction of the linear growth rate and an increase of the real frequency, relative to the collisionless limit. The effect of trapped particles under these conditions is to increase the growth rate and decrease the real frequency (i.e., to move it towards the ion diamagnetic direction). The dominant effect of the collisions, in turn, is to reduce the contribution of the trapped particles, accounting for the observed behavior.

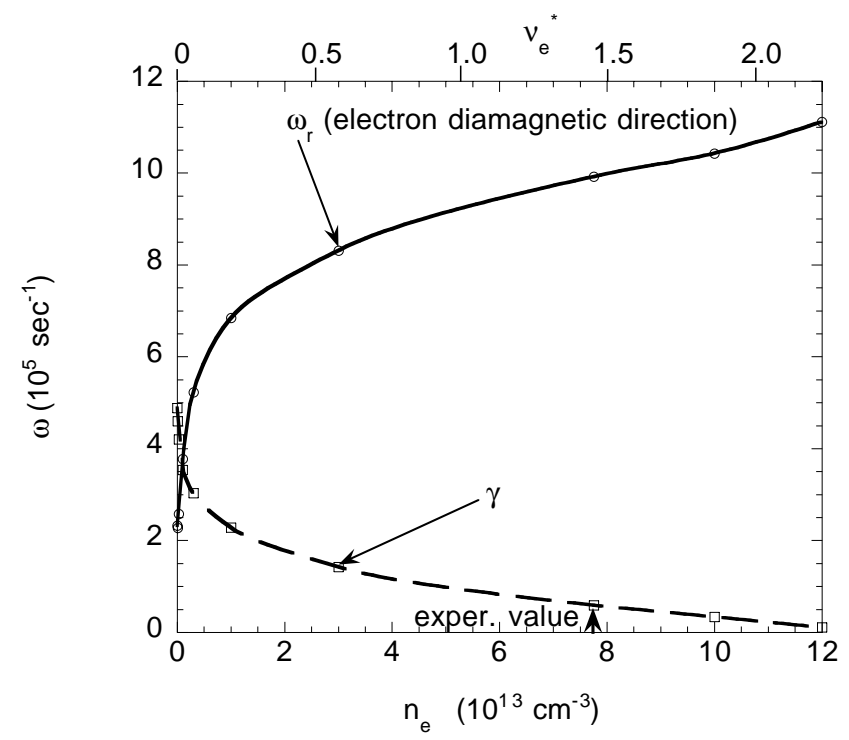

FIG. 9. Variation of growth rate $\gamma$ and real frequency $\omega_{r}$ with electron density $n_{e}$ and collisionality parameter $\nu_{e}^{*}$, for case of Fig. 4, for TEM root.

Finally, we perform a radial scan over the magnetic surface label $(s)$ for this case, including collisions. In the process, $k_{\perp} \rho_{i}(\theta=0)=0.56, \theta_{0}=0$, and $\alpha=0$ are kept fixed, and the other parameters are evaluated locally from the experimentally derived density and temperature profiles, so that the value of $n$ varies with $s$. The results for $\gamma$ are shown in Fig. 10(a) and for $\omega_{r}$ in Fig. 10(b). The TEM root is seen to be unstable only in the region 
$0.7 \lesssim s \lesssim 0.86$, which is a region of relatively strong density and temperature gradients. For discharges with different density and temperature profile shapes, the radial region of instability could be quite different. Note that in some nonlinear tokamak gyrokinetic simulations, it has been observed that the radial region of significant anomalous transport is significantly wider than the radial region of linear instability. ${ }^{16}$

\section{CONCLUSIONS}

The existing axisymmetric version of the linear microinstability code FULL was converted for nonaxisymmetric geometry, as described in Ref. 12. In the present experimentally-derived LHD case, two separate roots were found with this new version of FULL, one labeled as the trapped-electron mode (TEM) root, and one labeled as the ion temperature gradient mode (ITG) root which is only unstable above a critical value of the ion temperature gradient, with real frequencies in the electron and ion diamagnetic directions, respectively. For the experimental values of the electron and ion temperature gradients, only the TEM root is seen to be unstable. The maximum growth rate for the TEM root occurs for $k_{\perp} \rho_{i}(\theta=0)=0.5$ to 0.6 , as is also typical for tokamak cases. The maximum growth rate in the ballooning parameter $\theta_{0}$ and the field line label $\alpha \equiv \zeta-q \theta$ occurs for the TEM root for $\theta_{0}=\alpha=0$, a symmetry point of the MHD equilibrium. Collisions reduce the TEM root growth rate substantially, but it is still unstable for the experimental value of the density and collision frequency. Radially, the TEM root is unstable only over the range $0.7 \lesssim s \simeq(r / a)^{2} \lesssim 0.86$, but could have effects outside that region.

In Ref. 12, results for a quasiaxisymmetric case were given which were generally rather tokamak-like, in that there was only a small fraction of helically-trapped particles. In the

present LHD case, the fraction of helically-trapped particles is much larger, and the helicallytrapped particles and the helical curvature are seen to play a substantial destabilizing role for the TEM root. For the ITG root, on the other hand, their effect is moderately stabilizing. It is planned to investigate other cases for LHD also, in order to determine how general these 

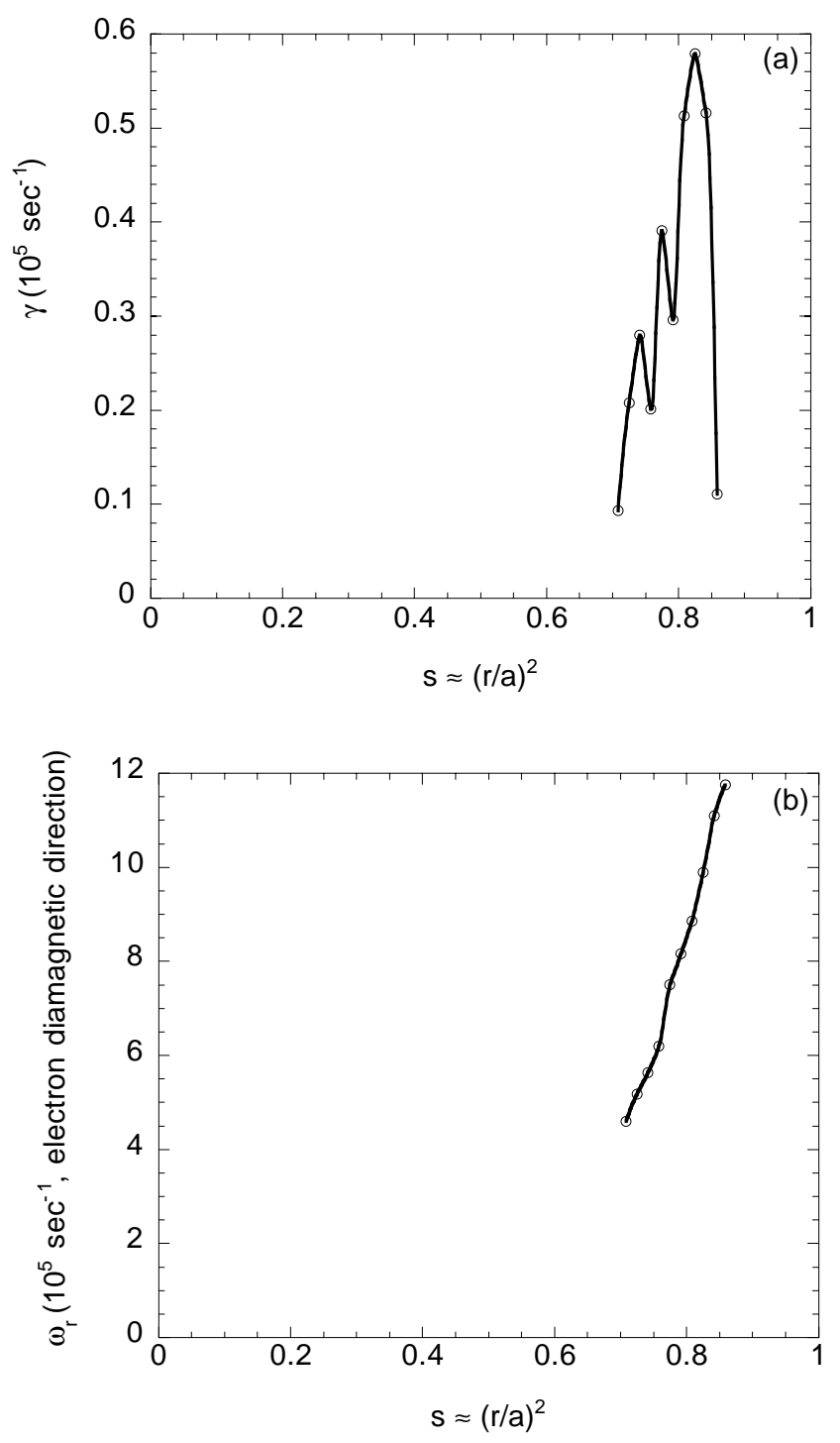

FIG. 10. Variation of (a) growth rate $\gamma$ and (b) real frequency $\omega_{r}$ with radial coordinate $s \simeq(r / a)^{2}$, for case of Fig. 4, for TEM root. 
trends are.

The only other work on microinstabilities in LHD of which we are presently aware is Ref. 17. The calculation there contains a number of approximations that are not made here. In Ref. 17, a simple model magnetic field is used to compare properties of ITG modes in tokamaks and in an LHD-like helical system, and it is argued that the negative magnetic shear and the helical ripples in the LHD-like configuration lower the growth rates of ITG modes by reducing the bad curvature on the outside of the torus. In that calculation, simplified dynamics with purely adiabatic electron response and no trapped particles and no collisions are used, while here the complete electron and trapped-particle response, along with a model collision operator, are kept in the calculation. Also, a different LHD case is considered there. Because of these differences, only rough qualitative agreement can be expected, and only for the ITG root. In fact, there is qualitative agreement on the values of $\alpha, \theta_{0}$, and $k_{\perp} \rho_{i}$ that maximize the growth rate, and also on the stabilizing effect of helical curvature, for this root.

In future work, other cases for LHD will be investigated, as well as additional cases resulting from the NCSX design effort. It would also be desirable to investigate cases for other stellarators that are currently in operation or are planned. In addition, evaluation of quasilinear particle and energy fluxes for all species, and extension to the fully electromagnetic case, are intended for the FULL code in future work.

\section{ACKNOWLEDGMENTS}

The authors would like to thank the LHD Experimental Group for fruitful communications. This work was supported by United States Department of Energy Contract No. DE-AC02-76-CHO-3073, and in part by a Grant-in-Aid from the Japanese Ministry of Education, Science, and Culture. 


\section{REFERENCES}

${ }^{1}$ A. Iiyoshi, A. Komori, A. Ejiri, et al., Plasma Physics and Controlled Nuclear Fusion Research, 1998, Nucl. Fusion 39, 1245 (1999).

${ }^{2}$ M. Fujiwara, H. Yamada, A. Ejiri, et al., Plasma Physics and Controlled Nuclear Fusion Research, 1998, Nucl. Fusion 39, 1659 (1999).

${ }^{3}$ S. P. Hirshman and D. K. Lee, Comput. Phys. Commun. 39, 161 (1986).

${ }^{4}$ S. P. Hirshman, U. Schwenn, and J. Nuehrenberg, J. Comput. Phys. 87, 396 (1990).

${ }^{5}$ A. H. Boozer, Phys. Fluids 24, 904 (1980).

${ }^{6}$ D. V. Anderson, W. A. Cooper, R. Gruber, S. Merazzi, and U. Schwenn, Int. J. Supercomp. Appl. 4, 34 (1990).

${ }^{7}$ W. Anthony Cooper, Plasma Phys. and Controlled Fusion 34, 1011 (1992).

${ }^{8}$ G. Rewoldt, W. M. Tang, and M. S. Chance, Phys. Fluids 25, 480 (1982).

${ }^{9}$ G. Rewoldt, W. M. Tang, and R. J. Hastie, Phys. Fluids 30, 807 (1987).

${ }^{10}$ A. Reiman, L. Ku, D. Monticello, et al., Plasma Physics and Controlled Nuclear Fusion Research, 1998, (International Atomic Energy Agency, Vienna, 1999), paper IAEA-CN-69/ICP/06, which is accessible on the web in PDF format at http://www.iaea.or.at/programmes/ripc/physics/html/node229.htm.

${ }^{11}$ A. Reiman, G. Fu, S. Hirshman, et al., Proceedings of the 26th European Physical Society Conference on Controlled Fusion and Plasma Physics Research, 1999, Plasma Phys. and Controlled Fusion 41, B273 (1999).

${ }^{12}$ G. Rewoldt, L.-P. Ku, W. M. Tang, and W. A. Cooper, Phys. Plasmas 6, 4705 (1999).

${ }^{13}$ S. Murakami, N. Nakajima, and M. Okamoto, Trans. Fusion Technol. 27, 256 (1995).

${ }^{14}$ B. Coppi and G. Rewoldt, in Advances in Plasma Physics, edited by A. Simon and W. B. 
Thompson (Wiley-Interscience, New York, 1976), Vol. 6, p. 421.

${ }^{15}$ M. Kotschenreuther, G. Rewoldt, and W. M. Tang, Comp. Phys. Comm. 88, 128 (1995).

${ }^{16}$ H. E. Mynick and S. E. Parker, Bull. Am. Phys. Soc. 41, 1359 (1996).

${ }^{17}$ T. Kuroda, H. Sugama, R. Kanno, and M. Okamoto, "Ion Temperature Gradient Modes in Toroidal Helical Systems", Report NIFS-632, to appear in J. Phys. Soc. Jpn. 
The Princeton Plasma Physics Laboratory is operated by Princeton University under contract with the U.S. Department of Energy.

\author{
Information Services \\ Princeton Plasma Physics Laboratory \\ P.O. Box 451 \\ Princeton, NJ 08543
}

Phone: 609-243-2750

Fax: 609-243-2751

e-mail: pppl_info@pppl.gov

Internet Address: http://www.pppl.gov 